\title{
Battery-aware Dynamical Modeling and Identification for the Total Thrust in Multi-rotor UAVs using only an Onboard Accelerometer
}

\author{
Nicolas Staub ${ }^{1,2}$ and Antonio Franchi ${ }^{1}$
}

\begin{abstract}
We propose and experimentally validate a new class of models for the total thrust generation in multi-rotor UAVs which is suitable for low- and middle-end platforms. Differently from typical models assuming to instantaneously control the rotor spinning velocity, in the proposed class we consider that the total thrust has its own dynamics and its final value explicitly depends both on the pseudo-setpoint commands given to the motor driver and the measurement of the battery terminal voltage. We compare the different model instances within the class using a principled experimental setup in which the total thrust is precisely measured using a motion capture system as ground truth, instead of relying on a setup based or noise-prone force sensors. We then show that the use of a dynamical model that includes also the battery terminal voltage significantly improves the prediction ability of the model in terms of accuracy. Finally we show how the proposed model can be identified using on-board only acceleration measurements, achieving a surprisingly good accuracy when compared with the ground truth case. We expect that the use of the proposed model will be important both in case of precise flight control and in the case of aerial physical interactive tasks.
\end{abstract}

\section{INTRODUCTION}

Unmanned Aerial Vehicles (UAVs) have been studied for decades, among those vehicles multi-rotor platforms raised recently the interest of the robotics research community for their agility, affordability and robust mechanical design. Typical multi-rotor platforms are actuated by a set of propellers with parallel rotational axes, whose number and spatial dispersion can vary among the platforms. This design is efficient in hovering, however it leads to underactuation, which must be addressed in the flight control.

Nowadays the aerial robotics community aims at enabling physical interaction for aerial systems like, e.g., for inspection [1] or assembly [2] tasks. To do so, control strategies have been proposed, based on different approaches. Nguyen et. Al. propose in [3] a hybrid force/position control, with a tangential motion control and a normal force control.Yüksel et. Al. [4] propose an Interconnection and Damping Assignment Passivity Based Control (IDA-PBC) scheme, where the total energy of the system is reshaped to mimic a target dynamics. Some other methods using more classical admittance [5] and impedance schemes [6] are also proposed in the literature. In this context the knowledge of the force exerted on the environment by the aerial robot is of paramount importance to achieve a precise interaction force control. Furthermore, it can be used also to retrieve external disturbance forces like, e.g., due to the wind.

\footnotetext{
${ }^{1}$ CNRS, LAAS, 7 avenue du colonel Roche, F-31400 Toulouse, France

${ }^{2}$ Univ de Toulouse, UPS, LAAS, F-31400 Toulouse, France nicolas.staub@laas.fr, antonio.franchi@laas.fr
}

To know the interaction force, one could either measure it directly at the point of contact or estimate it. The measurement approach relies on force sensors, whose ratio performance over weight and compactness is not suitable to be embedded in multi-rotors in the low/mid-cost range. Moreover to measure any force acting on the system by this mean, the sensor should be located at the interaction point, which is highly restricting for interaction applications and not possible for wind estimation. This leads to our choice of investigating an estimator based approach.

This observer should require low computation, and must be easy to reproduce, those two requirements are set to enforce usability of this method. The models used in the estimation process should remain as simple as possible to limit computational cost but still capture all the essential dynamics of the system in order to produce a trustworthy estimate. Once the models are set, their parameters estimation should be highly reproducible to be performed quickly after any modifications of the systems.

Estimation algorithms have been already studied in the literature. They mainly fall within two categories: force mappings and model based observers. A static mapping between commanded thrust and actual force has been proposed by [7] achieving a \pm 1 Newton accuracy. A more accurate, yet static, mapping of the force produced by a typical brushless motor for aerial vehicle has been proposed by [8], based on discretized force measurements for desired commands. In [9] a momentum based external generalized force observer is presented, which requires to measure the whole dynamical system state, the control torques and the thrust to produce an estimate of the external forces and torques. The methods has been proven to work indoor by using a precise off-board motion capture tracking. Yüksel et. al [10] proposed a non linear force observer, which requires to compute online both the position and attitude up to their 2nd derivatives. In [5] a classical Unscented Kalman Filter is presented to estimate the external force and torque acting on a quadrotor. This approach has also been tested with the use of an off-board motion capture system.

The main contribution of our work is to present a new kind of approach to estimate the total thrust produced by the propellers which is fast, computationally inexpensive and can be implemented only using onboard measurements (a singleaxis accelerometer), and without the knowledge of the multirotor state and/or it's derivatives. Contrarily to other existing static approaches [7], [8], [11] our approach is dynamical, since it takes into account the dynamics of the propeller in changing its rotational speed. In particular we propose a class of prediction models that use only the pseudo-setpoint of the 
propeller motor controller and the battery terminal voltage information, hereafter simply referred to as battery voltage. We conduct an experimental investigation to find the best and simplest model in this class using the acceleration retrieved from a motion capture system as ground-truth to validate our accelerometer-only-based approach. By comparing the prediction error for a set of possible inputs, we show that the addition of the battery voltage information in the model provides a manifest better force prediction. We alsot show experimentally that the usage of only onboard acceleration measurement for identification does not result in a significant degradation of the prediction when compared to the use of ground truth for the same purpose.

The rest of the paper is organized as follows. Section II presents our proposed class of models for the force generation. Those models are proposed based on observations made in the literature or on our test bed. In Section III the method to estimate the parameters of the aforementioned models are derived. Section IV describes the experimental set up that we used to acquire the data and Sec. V presents the results of the experiment analysis with respect to our claims. Finally Section VI presents a summary of the presented work and ongoing developments.

\section{THE PROPOSED MODEL CLASS}

In this section we extend the classical model of an underactuated multi rotor vehicle in order to include the presence of an unknown motor-driver dynamics for each propeller. We first start by recalling the standard model.

\section{A. Multi-rotor Vehicle Dynamics and Standard Motor Model}

We denote with $\{A\}$ the inertial frame whose origin is $O_{A}$ and unit vectors along the axes are denoted by $\left\{\overrightarrow{\mathbf{a}}_{1}, \overrightarrow{\mathbf{a}}_{2}, \overrightarrow{\mathbf{a}}_{3}\right\}$. The vector $\mathbf{p} \in \mathbb{R}^{3}$ represents the position of the Center of Mass (CoM) of the aerial vehicle expressed in $\{A\}$. Denote with $\{B\}$ the body fixed frame whose origin coincides with the CoM of the vehicle, and axes w.r.t. $\{A\}$ are represented by the unit vectors $\left\{\overrightarrow{\mathbf{b}}_{1}, \overrightarrow{\mathbf{b}}_{2}, \overrightarrow{\mathbf{b}}_{3}\right\}$. We assume, as standard in the aeronautic field, that $\{B\}$ is oriented with the NorthEast-Down (NED) convention, i.e., $\overrightarrow{\mathbf{b}}_{3}$ is pointing downwards when the aerial vehicle is hovering. The orientation of the vehicle in $\{A\}$ is provided by the rotation matrix ${ }^{A} R_{B}=R=$ $\left(\overrightarrow{\mathbf{b}}_{1} \overrightarrow{\mathbf{b}}_{2} \overrightarrow{\mathbf{b}}_{3}\right)[12]$.

Denote with $N$ the number of rotors of the vehicle, which are all spinning on the plane defined by $\overrightarrow{\mathbf{b}}_{1}$ and $\overrightarrow{\mathbf{b}}_{2}$, thus about $\overrightarrow{\mathbf{b}}_{3}$. The equation of motion of the vehicle's CoM can be written as:

$$
m \ddot{\mathbf{p}}=m g \overrightarrow{\mathbf{a}_{3}}+R \mathbf{f},
$$

where $m \in \mathbb{R}_{+}$is the mass of the vehicle, $g \overrightarrow{\mathbf{a}_{3}}$ is the gravity acceleration, and $\mathbf{f} \in \mathbb{R}^{3}$ is the total force acting on the vehicle, expressed at the center of gravity in $\{B\}$. The dominant aerodynamics of each rotor $i$, for $i=1, \ldots, N$, produces a force (thrust) $F_{i} \overrightarrow{\mathbf{b}_{3}}$ (see, e.g., [12]). In contactfree flight, the total force, expressed in body frame, is then

$$
\mathbf{f}=\sum_{i=1}^{N} F_{i} \overrightarrow{\mathbf{b}_{3}}+\delta=F_{T} \overrightarrow{\mathbf{b}_{3}}+\delta
$$

where

$$
F_{T}=\sum_{i=1}^{N} F_{i}
$$

is the total thrust, and $\delta$ comprises second order aerodynamic forces mainly due to flapping and drag effects, that are typically neglected in nominal working conditions [12]. It can be assumed [12] that the thrust produced by the rotor $i$ is instantaneously related to its spinning velocity $\varpi_{i}$ by the following relation

$$
F_{i}=c_{F} \varpi_{i}^{2}
$$

where $c_{F}>0$ is an aerodynamic constant that depends on the specific properties of the propeller used.

\section{B. Possible Drawbacks of the Standard Model}

Many of the previous works, see e.g. [12], assume that $\varpi_{i}$ is the control input of the system and that $c_{F}$ is known. Under these two assumptions the $F_{i}$ for $i=1 \ldots N$ can be considered as control inputs, which makes the control problem simpler. However, these assumptions are hard to be met in reality for the reasons that are explained in the following. First of $c_{F}$ is in many cases unknown, depends on the propeller type, and even vary within the same type because of normal large-scale production variations. Second of all the motor has its own dynamics. Therefore $\varpi_{i}$ cannot be changed instantaneously acting on the torque applied by the motor driver.

In fact, in the best case, the motor control input is usually a setpoint for $\bar{\varpi}_{i}$, which is tracked by the motor driver with a certain dynamics and accuracy. The control accuracy of $F_{i}$ depends then on the accuracy in the knowledge of the parameter $c_{F}$ (needed in order to generate the setpoint for the motor driver) and on the accuracy of the driver in tracking the velocity setpoint. For typical multi-rotor platforms, especially in the low/mid-cost range it is not possible to send a setpoint for the spinning velocity to the motor drivers, but rather a pseudo-setpoint, e.g., a PWM signal, which is monotically related to the steady state spinning velocity, if all the other flight conditions are constant.

In both cases (for setpoint and for pseudo-setpoint) the battery voltage level has an impact on the behavior of the motor controller, as noted, e.g., in [11]. In particular, the battery voltage decreases as long as the battery discharges due to the increase of the internal resistance. This measurement can be found among all the type of platforms. During contactfree flight, the dependence to the battery voltage can be overcome by using an adaptive term in the control law which compensates the discharge of the battery. Such a method can take the form of a mass estimator or an integral term [8], [13], which increases the average of the commands sent to the motors, thus compensating the voltage drop. This approach is working because the only force acting on the system are the weight and the thrust generated by the propellers. For physical interaction this technique can not be used since a force sensor is unavailable in our assumptions and the interaction force involved in the balance is typically unknow.

In order to have a good control of the force exerted by the system it is instead important to have control on the 


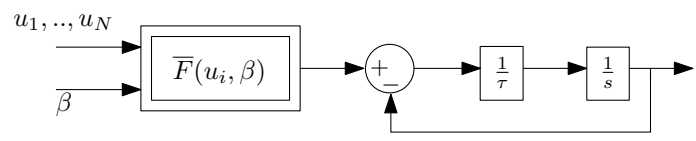

Fig. 1: Block diagram of the proposed model

total thrust $F_{T}$ exerted by the rotors. Therefore another viable approach, considered in this paper, is to take into account the battery voltage influence and the motor dynamics directly in the input-output nonlinear model. Since we are interested in controlling the force we try to identify the direct relation between the (pseudo)-setpoints and the battery voltage as inputs and the force as output.

\section{Model Based on (Pseudo)-Setpoint and Battery Level}

Denote with $u_{i} \in\left[u_{\min }, u_{\max }\right]$, with $u_{\min }>0$ the control input of the motor driver, which may represent either a setpoint or a pseudo-setpoint for the driver of the $i$ motor of the vehicle. Denote with $\beta$ the battery voltage level, that we assume to be measured. Our goal is then to propose a modeling of the relation between $u_{i}$ and $\beta$ and $F_{i}$ that is as simple as possible but captures all the relevant dynamics.

Based on the analysis of experimental data, see e.g., [8], [11], we have made the following observations:

O1. if $u_{i}$ is kept constant for a time window of the order of magnitude of $\approx 1$ second then $F_{i}$ reaches a constant value that is is monotonically increasing w.r.t. $u_{i}$ and monotonically decreasing w.r.t. $\beta$.

Motivated by the previous observation we propose the following simple model for the force

$$
\dot{F}_{i}=\frac{1}{\tau_{i}}\left(\bar{F}_{i}\left(u_{i}, \beta\right)-F_{i}\right)
$$

where $\bar{F}\left(u_{i}, \beta\right)$ is an unknown nonlinear map, and $\tau_{i}$ is an unknown time constant, this model can be represented as a nonlinear block and a linear first order system, see Fig.1.

In order to provide a simple expression of $F_{T}$ let us consider the following reasonable assumptions:

A 1. $\bar{F}_{i}\left(u_{i}, \beta\right)$ and $\tau_{i}$ are the same across all motor controllers, i.e., for $i=1 \ldots N$

A 2. $\bar{F}_{i}\left(u_{i}, \beta\right)$ is a smooth function that can be well approximated by a finite polynomial of a suitable order in the region of interest $u_{i} \in\left[u_{\min }, u_{\max }\right]$ and $\beta \in\left[\beta_{\min }, \beta_{\max }\right]$

Given Assumptions 1 and 2 we write

$$
\begin{array}{clrl}
\tau_{i} & =\tau, & & \forall i=1 \ldots N \\
\bar{F}_{i}\left(u_{i}, \beta\right) & =\bar{F}\left(u_{i}, \beta\right) \approx \sum_{j=0}^{n_{u}} \sum_{k=0}^{n_{\beta}}\left(\alpha_{j k} u_{i}^{j} \beta^{k}\right), & & \forall i=1 \ldots N
\end{array}
$$

where $n_{u}, n_{\beta} \in \mathbb{N}$ have to be chosen taking into account the desired model complexity, and $\alpha_{j k}$, with $j=1 \ldots n_{u}, k=$ $1 \ldots n_{\beta}$, are $n_{u} \cdot n_{\beta}$ parameters to be estimated.

Using (3),(5),(6),(7) we can write the dynamics of the total thrust $F_{T}$ as

$$
\begin{aligned}
\dot{F}_{T} & \approx \sum_{i=1}^{N} \frac{1}{\tau_{i}}\left(\sum_{j=0}^{n_{u}} \sum_{k=0}^{n_{\beta}}\left(\alpha_{j k} u_{i}^{j} \beta^{k}\right)-F_{i}\right) \\
& =\frac{1}{\tau}\left(\sum_{j=0}^{n_{u}} \sum_{k=0}^{n_{\beta}}\left(\alpha_{j k} \beta^{k} \sum_{i=1}^{N} u_{i}^{j}\right)-F_{T}\right) \\
& =\frac{1}{\tau}\left(\sum_{j=0}^{n_{u}} \sum_{k=0}^{n_{\beta}} \alpha_{j k} v_{j k}-F_{T}\right)
\end{aligned}
$$

where we compactly wrote $v_{j k}=\beta^{k} \sum_{i=1}^{N} u_{i}^{j}$.

The equation (10) is describing a class of sufficiently simple nonlinear dynamical models for the total thrust of a multi-rotor platform using only the (pseudo)-setpoint and the battery voltage, both information that can bee found on nearly every, if not all, platforms. This class has been introduced in order to illustrate two of our assertions which constitute part of our contribution:

Claim 1. Using only (pseudo)-setpoint and battery voltage in the model, it is possible to predict the actual total thrust with a 'good' confidence.

Claim 2. Furthermore the use of the battery voltage information improves 'substantially' the quality of the prediction.

The model class size is only limited by the choice of $n_{u}$ and $n_{\beta}$, among the models present in the class some have better performance than others. Our goal is to find the simplest model (i.e., the one with the lowest number of parameters) that provides a sufficient prediction performance compared to more complex models.

\section{IDENTIFICATION OF MODEL PARAMETERS}

Model (10) represents a class of models, depending on the values of the parameters $n_{u}, n_{\beta}$. In this class of models, three quantities are needed in order to estimate the parameters $\tau$ and the $\alpha_{j k}$ 's: the model inputs, i.e., the (pseudo)-setpoint $u_{i}$ and the battery voltage $\beta$, and the model's output, i.e., the total thrust $F_{T}$. The information on $F_{T}$ can be replaced by acceleration information, given that the mass $m$ of the system is known. In practical situations two cases, detailed in the following, can happen.

The case in which $\ddot{\mathbf{p}}$ can be precisely measured (e.g., using a motion capture system). In this case the thrust force in the inertial frame, ${ }^{A} \mathbf{f}=R \mathbf{f}$, can be computed by directly employing (1). The total thrust $F_{T}$ is then computed as $\left\|{ }^{A} \mathbf{f}\right\|$. We shall use this case as ground truth in the experiments.

The second case is when the vehicle is equipped with a calibrated IMU which measures the proper acceleration of the vehicle expressed in the body frame $\{B\}$, i.e.,

$$
\overline{\mathbf{a}}=R^{T}(\ddot{\mathbf{p}}-g \overrightarrow{\mathbf{z}})+\eta_{I M U},
$$

where $\eta_{I M U}$ is some additive noise with zero mean. This case will be used in the experiments to show that the model can be effectively identified using only onboard measurements. Using (1),(2) in (11) we obtain

$$
\overline{\mathbf{a}}=\frac{F_{T}}{m} \overrightarrow{\mathbf{z}}+\frac{1}{m} \delta+\eta_{I M U} .
$$




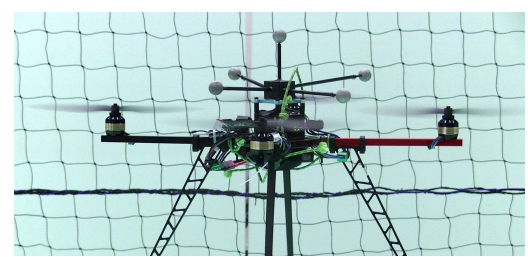

Fig. 2: The multi-rotor platform used at LAAS

In typical conditions (i.e., at low speed and at a certain distance from the ground ) $F_{T}$ is much larger than the third component of $\|\delta\|$. Therefore, we can write

$$
F_{T}=m \overrightarrow{\mathbf{z}}^{T} \overline{\mathbf{a}}+\eta_{F} \approx m \bar{a}_{z} .
$$

Where $\eta_{f}$ represents a negligible contributions of both the aerodynamic effects and the IMU noise. Assuming that the mass $m$ of the vehicle can be measured before the flight, we shall use (13) as an onboard measurement of the total thrust. In particular, we make the following claim that will be tested in the experimental section:

Claim 3. The readings of calibrated low/middle-cost accelerometers are sufficiently accurate to perform a reliable identification of the parameters of the proposed model, leading to practically acceptable onboard total thrust prediction.

\section{A. Use of Estimated Model in Contact Tasks}

Notice that (13) is valid only in contact-free flight, i.e., when the non-gravitational forces acting on the vehicle can be expressed by (2). During contact, (2) includes also the interaction forces $\mathbf{f}_{e}$, thus it can be rewritten as

$$
m \ddot{\mathbf{p}}=m g \overrightarrow{\mathbf{a}_{3}}+R \mathbf{f}+\mathbf{f}_{e} .
$$

The estimation of $F_{T}$ can be performed in the same way as before if $\mathbf{f}_{e}$ is known.

If instead the estimation of $\tau$ and $\bar{F}$ is performed using measurements taken during contact-free flight phases, the identified model can then be used to estimate $\mathbf{f}_{e}$ in contact phases since both $\tau$ and $\bar{F}$ are constant over time. We leave the validation of this claim as a future work. while in the following we provide the method to estimate the parameters of the force model.

\section{EXPERIMENT DESIGN}

In order to estimate the parameters in (10) we designed an experimental set up based on the Telekyb framework [13] and composed by a motion capture system (mocap), a control computer and a quadrotor, see Fig. 2. The mocap provides the tracked object position at $100 \mathrm{~Hz}$ with millimeter-scale accuracy. A very accurate estimation of the velocity and acceleration is obtained post-processing the measured position with a non-causal Savitzky-Golay filter [14].

The quadrotor mechatronics is based on the Mikrokopter platform, whereas the flight control software has been replaced by the Telekyb [13] one. Two Xbee radio transmitters are used to send commands to the quadrotor and to record the telemetry data, respectively. The data recorded are: the battery voltage, a timer for synchronization, the four motor pseudo-setpoints (PWM signals) sent by the flight controller to the brushless controllers and the onboard acceleration measurements provided by the threee 3D LIS344alh accelerometers $\left(0.0057 g_{0} \mathrm{~m} / \mathrm{s}^{2}\right.$ resolution and $\pm 2 g_{0} \mathrm{~m} / \mathrm{s}^{2}$ range). The accelerometers are calibrated following the procedure detailed in [8]. The control computer runs the Telekyb control framework under the middleware ROS. The flight controller used for the quadrotor is a near-hovering scheme which allows to follow trajectories where the roll and pitch of the quadrotor remain within $\pm 30^{\circ}$.

The thrust is then computed twice using $(i)$ the acceleration measurement from the motion capture (ground truth) and (ii) the accelerometer reading, as explained in Sec. III.

Any trajectory that spans the the battery range and the pseudo-setpoint range of interest can be used for the identification. The former requirement asks for a flight duration that discharges the battery enough and the latter one asks for a sufficiently rich acceleration content of the tracked trajectory. To meet the arena-size constraint as well, a vertical trajectory has been chosen providing enough space to reach high accelerations while remaining near the hovering attitude. To ensure the spectral richness of the acceleration the vertical trajectory is composed of five sinusoids with different pulsations:

$$
z(t)=\rho+\sum_{i=1}^{5} a_{i} \sin \left(\omega_{i} t+\phi_{i}\right)
$$

where $\rho=2$ is a position offset and

$$
\begin{aligned}
{\left[\omega_{1}, \ldots, \omega_{5}\right] } & =2 \pi \cdot[0.3,0.2,0.1,0.5,0.6] \\
{\left[a_{1}, \ldots, a_{5}\right] } & =0.3 \cdot\left[\omega_{1}^{-2}, 0.95 \omega_{2}^{-2}, 0.9 \omega_{3}^{-2}, 0.8 \omega_{4}^{-2}, 0.7 \omega_{5}^{-2}\right] \\
\phi & =\pi \cdot\left[\frac{1}{2}, \frac{1}{3}, \frac{1}{4}, \frac{1}{5}, \frac{1}{6}\right]
\end{aligned}
$$

are the pulsations, the amplitudes, and the phase shifts of the sinusoids, respectively. With this choice the input is then persistently exciting with order 10, making us able to identify of up to 10 parameters in (10), see, e.g., [16].

\section{EXPERIMENTAL RESULTS}

To estimated the parameters of (10) we used a predictive error method coupled with a grey-box model. This method is a numerical optimization with a cost function based on the norm of the prediction [15]. Three different analysis have been conducted on the data and are described hereafter.

In order to illustrate the experimental setup, the video attached to the paper shows a preliminary experiment with a simpler trajectory.

\begin{tabular}{ccccccccccc}
\hline ID of the model & 1 & 2 & 3 & 4 & 5 & 6 & 7 & 8 & 9 & 10 \\
\hline$n_{u}$ & 0 & 0 & 1 & 1 & 0 & 2 & 1 & 2 & 2 & 2 \\
\hline$n_{\beta}$ & 0 & 1 & 0 & 1 & 2 & 0 & 2 & 1 & 2 & 2 \\
\hline Numb. of param. & 2 & 3 & 3 & 4 & 4 & 5 & 7 & 7 & 10 & 10 \\
\hline
\end{tabular}

TABLE I: Different models considered in the identification. 


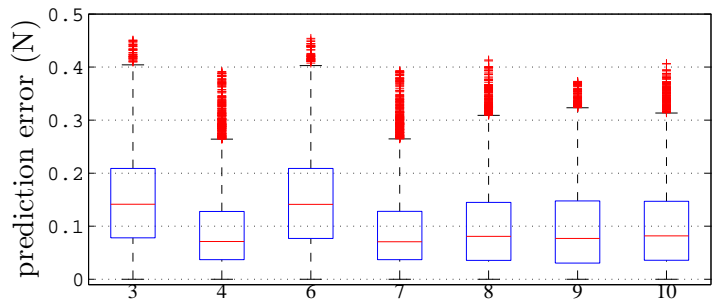

Fig. 3: Boxplot of the prediction error for the proposed models (see Tab. I), the parameters are estimated with the ground truth and the prediction is compared against the ground truth.

\begin{tabular}{cccccccc}
\hline & model_3 & model_4 & model_6 & model_7 & model_8 & model_9 & model_10 \\
\hline$\tau$ & 0.011 & 0.019 & 0.011 & 0.019 & 0.013 & 0.039 & 0.016 \\
\hline$\alpha_{00}$ & $-2.234 \mathrm{e}-01$ & $-8.895 \mathrm{e}-01$ & $-2.632 \mathrm{e}-01$ & 6.610 & -1.843 & $-1.359 \mathrm{e}+02$ & $1.556 \mathrm{e}+02$ \\
\hline$\alpha_{01}$ & - & $3.805 \mathrm{e}-05$ & - & $-6.194 \mathrm{e}-04$ & $7.564 \mathrm{e}-05$ & $1.177 \mathrm{e}-02$ & $-1.375 \mathrm{e}-02$ \\
\hline$\alpha_{02}$ & - & $1.080 \mathrm{e}-04$ & $-3.200 \mathrm{e}-05$ & $-6.487 \mathrm{e}-04$ & $3.091 \mathrm{e}-04$ & $4.438 \mathrm{e}-02$ & $-2.094 \mathrm{e}-02$ \\
\hline$\alpha_{10}$ & $-4.056 \mathrm{e}-05$ & $-7.484 \mathrm{e}-09$ & - & $5.930 \mathrm{e}-08$ & $-1.579 \mathrm{e}-08$ & $-3.894 \mathrm{e}-06$ & $1.864 \mathrm{e}-06$ \\
\hline$\alpha_{11}$ & - & - & - & $1.440 \mathrm{e}-08$ & - & $-2.551 \mathrm{e}-07$ & $3.034 \mathrm{e}-07$ \\
\hline$\alpha_{12}$ & - & - & $-4.035 \mathrm{e}-10$ & - & $-1.173 \mathrm{e}-08$ & $-2.701 \mathrm{e}-06$ & $4.646 \mathrm{e}-07$ \\
\hline$\alpha_{20}$ & - & - & - & $-1.473 \mathrm{e}-12$ & - & $8.527 \mathrm{e}-11$ & $-4.157 \mathrm{e}-11$ \\
\hline$\alpha_{21}$ & - & - & - & - & $5.062 \mathrm{e}-13$ & $2.373 \mathrm{e}-10$ & $-4.168 \mathrm{e}-11$ \\
\hline$\alpha_{22}$ & - & - & - & - & - & $-5.214 \mathrm{e}-15$ & $9.337 \mathrm{e}-16$ \\
\hline
\end{tabular}

TABLE II: Recapitulative table of the estimated parameters of the models when the estimation is conducted with mocap data.

\section{A. Model Order Choice and Mocap-based Identification}

The number of parameters in (10) is $\left(n_{u}+1\right) \cdot\left(n_{\beta}+1\right)+$ 1 , thus it depends on the chosen value of $n_{u}$ and $n_{\beta}$. Our goal is to find $n_{u}$ and $n_{\beta}$ such that the system dynamics is well described by the estimated model but with the smallest number of parameters.

As explained in Sec. IV the maximum number of parameters that we can identify with our experimental setup is 10 . Therefore we can consider $n_{u}, n_{\beta} \in\{0,1,2\}$. The 10 resulting models are summarized in Table I, where models 9 and 10 have the same structure but the parameters are estimated with a different initial guess as explained in the following. We discard model 1 since the system dynamics can not be described just by a constant. Models 2 and 5 are also discarded as they do not contain information on the (pseudo)-setpoint. The initial guess for the estimation of the parameters of more complex models is provided by the solution of the antecedent in the model class, thus creating the following orders: $3 \rightarrow 4 \rightarrow 7 \rightarrow 9$ and $3 \rightarrow 6 \rightarrow 8 \rightarrow 10$.

We first estimated the parameters using a set of data from the mocap (we shall present the estimation using onboardonly sensor in Sec. V-C). The estimated parameters are then validated against another set of data from the mocap. To better validate the ability of prediction of our models w.r.t. the battery effect, the battery voltages in the 2 sets span completely different values. Boxplots graphs of the absolute value from the prediction error are presented in Fig. 3, the predictions are quite good overall, with a prediction error median around or under $0.1 \mathrm{~N}$, which is remarkable considering that the range of the recorded force during the experiment is $[7.5 \mathrm{~N}, 12.2 \mathrm{~N}]$. This demonstrates that the

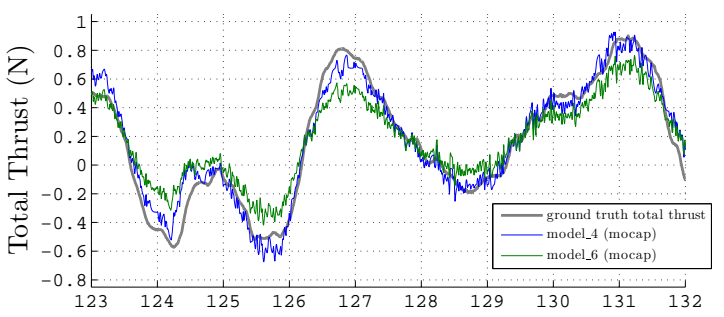

Fig. 4: Comparison of two estimated model one not including battery info (model 6) and one including battery info (model 4). Both models have the same number of parameters, the one containing battery information is clearly more performant.

proposed class of models is able to predict the total thrust accurately thus validating our first claim. The complete set of estimated parameters is presented in Table II. Notice that the time constant is of the same magnitude for all the models, with model 9 having a slightly higher one.

\section{B. Discussion on Battery Influence}

From the previous model perfomances one can investigate the importance of the battery. Models 3 and 6, where battery voltage information is not used, are both outperformed (higher median, wider dispersion) by the models containing both pseudo-setpoint and battery information, i.e., 4, 7, 8, 9, and 10. As models 3 and 4 have the same number of inputs the use of battery data is the only changing factor that can explain the better fitting of 4 with respect to 3 . Moreover, we can notice that despite of the fact that model 6 has more inputs than model 4 , its prediction ability is worse. In general we can conclude that the models in which $n_{\beta} \neq 0$ can better predict the system behavior by taking into account the battery voltage drop along the flight. This fact removes, e.g., the need for an adaptation term in the flight controller. This result validate our second claim, i.e., that the use of the battery voltage information improves 'substantially' the quality of the prediction. A direct comparison between model 4 and 6 on a chunk of the validation set is shown in Fig. 4.

\section{Accelerometer-based Identification}

An analysis was conducted to determine the validity of parameters estimation based only on the onboard accelerometer measurements. Then the quality of the prediction of those models has been compared to ground truth (i.e., the validation set used in the mocap case). The prediction error against the mocap is presented in Fig. 5.

The comparison of Fig. 3 and Fig. 5 shows that the parameter estimation process can also be conducted without a mocap at the cost of minimal variation of the prediction error, allowing the proposed force prediction framework to be deployed without a mocap. This result validates our third claim, i.e., that the use of a calibrated low/middle-cost accelerometer is sufficient to perform a reliable identification of the parameters of the proposed model, leading to practically acceptable onboard total thrust prediction. The complete set of estimated parameters can be found in Table III. 


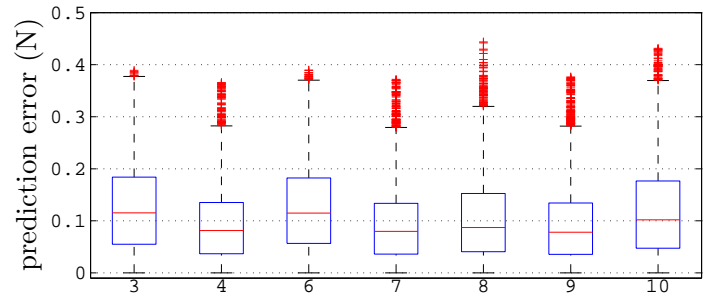

Fig. 5: Boxplot of the prediction error for the proposed models (see Tab. I), the parameters are estimated with onboard acceleration and the prediction is compared against the ground truth

\begin{tabular}{cccccccc}
\hline & model_3 & model_4 & model_6 & model_7 & model_8 & model_9 & model_10 \\
\hline$\tau$ & 0.013 & 0.016 & 0.014 & 0.015 & 0.003 & 0.014 & 0.003 \\
\hline$\alpha_{00}$ & $-1.774 \mathrm{e}-01$ & $-4.144 \mathrm{e}-01$ & $-2.370 \mathrm{e}-01$ & $1.708 \mathrm{e}+01$ & $-4.815 \mathrm{e}-02$ & $-4.025 \mathrm{e}-01$ & $3.376 \mathrm{e}+02$ \\
\hline$\alpha_{01}$ & - & $1.729 \mathrm{e}-05$ & - & $-1.515 \mathrm{e}-03$ & $-6.279 \mathrm{e}-07$ & $2.493 \mathrm{e}-05$ & $-2.960 \mathrm{e}-02$ \\
\hline$\alpha_{02}$ & - & $4.287 \mathrm{e}-05$ & $-3.224 \mathrm{e}-05$ & $-1.677 \mathrm{e}-03$ & $-3.365 \mathrm{e}-05$ & $4.692 \mathrm{e}-03$ & $-4.748 \mathrm{e}-02$ \\
\hline$\alpha_{10}$ & $-4.503 \mathrm{e}-05$ & $-4.601 \mathrm{e}-09$ & - & $1.470 \mathrm{e}-07$ & $-8.840 \mathrm{e}-10$ & $-4.164 \mathrm{e}-07$ & $4.185 \mathrm{e}-06$ \\
\hline$\alpha_{11}$ & - & - & - & $3.352 \mathrm{e}-08$ & - & $-3.756 \mathrm{e}-10$ & $6.485 \mathrm{e}-07$ \\
\hline$\alpha_{12}$ & - & - & $-6.025 \mathrm{e}-10$ & - & $3.192 \mathrm{e}-09$ & $-4.036 \mathrm{e}-07$ & $1.199 \mathrm{e}-06$ \\
\hline$\alpha_{20}$ & - & - & - & $-3.338 \mathrm{e}-12$ & - & $9.119 \mathrm{e}-12$ & $-9.228 \mathrm{e}-11$ \\
\hline$\alpha_{21}$ & - & - & - & - & $-1.541 \mathrm{e}-13$ & $3.568 \mathrm{e}-11$ & $-1.055 \mathrm{e}-10$ \\
\hline$\alpha_{22}$ & - & - & - & - & - & $-7.882 \mathrm{e}-16$ & $2.322 \mathrm{e}-15$ \\
\hline
\end{tabular}

TABLE III: Recapitulative table of the estimated parameters of the models when the estimation is conducted with accelerometers data.

\section{Choice of the Best Model}

From our analysis, model 4 results to be the best compromise between accuracy and complexity. In fact, this model resulted able to describe the dynamics of the system with the same level of prediction ability of models with more parameters. Furthermore, model 4 is computationally lightweight and therefore can be easily implemented, e.g., on a microcontroller. Furthermore, this model keeps substantially the same the prediction ability if the estimation of the parameters is conducted using onboard accelerometer data.

\section{CONCLUSIONS}

In this work we proposed a class of dynamic models to predict the force (total thrust) generated by a underactuated multi-rotor system. The major contributions have been to consider the motor dynamics, include battery voltage information in the prediction model, and to identify the model parameters using only onboard accelerometer measurements. We conducted an experimental investigation to find the best model among the simplest ones in this class using the acceleration retrieved from mocap measurements as groundtruth.

By comparing the prediction error for a set of possible inputs, we have shown that the addition of the battery voltage information in the model provides a manifest better force prediction. From our experimental result it was also clear that the usage of only onboard acceleration measurement for identification does not result in a significant degradation of the prediction when compared to the use of ground truth for the same purpose.

Nevertheless the assumption is made that the only force acting on the system during the record of the data for parameter estimation are the weight force and the total thrust, thus an outdoor estimation of the parameters in windy conditions is not possible. However, the proposed models can be used for an outdoor estimation of external forces acting on the system, like e.g., wind.

In the near future we are working toward the integration of this work in a physical interaction framework, where we want to use the force prediction to close the control loop on external forces.

\section{REFERENCES}

[1] AIRobots, "EU Collab. Project ICT-248669," www.airobots.eu.

[2] ARCAS, "EU Collab. Project ICT-287617," www.arcas-project.eu.

[3] H. Nguyen and D. Lee, "Hybrid force/motion control and internal dynamics of quadrotors for tool operation," in 2013 IEEE/RSJ Int Conf. on Intelligent Robots and Systems, Tokyo, Japan, November 2013, pp. 3458-3464.

[4] B. Yüksel, C. Secchi, H. H. Bülthoff, and A. Franchi, "Reshaping the physical properties of a quadrotor through IDA-PBC and its application to aerial physical interaction," in 2014 IEEE Int. Conf. on Robotics and Automation, Hong Kong, China, May. 2014, pp. 62586265 .

[5] F. Augugliaro and R. DAndrea, "Admittance control for physical human-quadrocopter interaction," in 12th European Control Conference, Zurich, Switzerland, Jul. 2013, pp. 1805-1810.

[6] G. Gioioso, M. Ryll, D. Prattichizzo, H. H. Bülthoff, and A. Franchi, "Turning a near-hovering controlled quadrotor into a 3D force effector," in 2014 IEEE Int. Conf. on Robotics and Automation, Hong Kong, China, May. 2014, pp. 6278-6284.

[7] S. Bellens, J. De Schutter, and H. Bruyninckx, "A hybrid pose/wrench control framework for quadrotor helicopters," in 2012 IEEE Int. Conf. on Robotics and Automation, St.Paul, MN, May 2012, pp. 2269-2274.

[8] R. Spica, P. Robuffo Giordano, M. Ryll, H. H. Bülthoff, and A. Franchi, "An open-source hardware/software architecture for quadrotor UAVs," in 2nd IFAC Work. on Research, Education and Development of Unmanned Aerial Systems, Compiegne, France, Nov. 2013.

[9] F. Ruggiero, J. Cacace, H. Sadeghian, and V. Lippiello, "Impedance control of VToL UAVs with a momentum-based external generalized forces estimator," in 2014 IEEE Int. Conf. on Robotics and Automation, Hong Kong, China, Apr. 2014, pp. 2093-2099.

[10] B. Yüksel, C. Secchi, H. H. Bülthoff, and A. Franchi, "A nonlinear force observer for quadrotors and application to physical interactive tasks," in 2014 IEEE/ASME Int. Conf. on Advanced Intelligent Mechatronics, Besançon, France, Jul. 2014, pp. 433-440.

[11] I. Sa and P. Corke, "System identification, estimation and control for a cost effective open-source quadcopter," in 2012 IEEE Int. Conf. on Robotics and Automation, May 2012.

[12] R. Mahony, V. Kumar, and P. Corke, "Multirotor Aerial Vehicles: Modeling, Estimation, and Control of Quadrotor," IEEE Robotics \& Automation Magazine, vol. 19, no. 3, pp. 20-32, 2012.

[13] V. Grabe, M. Riedel, H. H. Bülthoff, P. Robuffo Giordano, and A. Franchi, "The TeleKyb framework for a modular and extendible ROS-based quadrotor control," in 6th European Conference on Mobile Robots, Barcelona, Spain, Sep. 2013, pp. 19-25.

[14] A. Savitzky, , and M. J. E. Golay, "Smoothing and differentiation of data by simplified least squares procedures." Analytical Chemistry, vol. 36, no. 8, pp. 1627-1639, 1964

[15] T. S. Soderstrom and P. G. Stoica, System Identification, ser. Prentice Hall International Series In Systems And Control Engineering. Prentice Hall, 1989.

[16] L. Ljung, System Identification - Theory For the User, 2nd ed. Upper Saddle River, N.J.: PTR Prentice Hall, 1999. 